\title{
A prospective study of OAR volume variations between two different treatment planning systems in radiotherapy
}

\author{
Bhudevi Soubhagya Kulkarni', Sunil Dutt Sharma ${ }^{2}$, Vibeka Hansen ${ }^{3}$, NVN Madhusudhana Sresty', \\ Suneetha M1, Mani Kandan ${ }^{4}$, Chandrashekhar D ${ }^{5}$, Anil Kumar Talluri ${ }^{1}$, Alok Kumar', Shabbir Ahmed ${ }^{1}$ \\ ${ }^{I}$ Department of Radiation Oncology, Basav tarak indo American cancer hospital and research centre, Hyderabad, India \\ ${ }^{2}$ Department of Radiological Physics \& Advisory Division, Bhabha Atomic Research Centre, \\ CTCRS, Anushaktinagar, Mumbai, India \\ ${ }^{3}$ Joint department of Physics, Royal Marsden NHS Trust and The Institute of Cancer Research, Sutton, United Kingdom \\ ${ }^{4}$ Department of Radiation Oncology, NRI hospital, Guntur, India \\ ${ }^{5}$ Department of Physics, Jawaharlal Nehru Technological University, Hyderabad, India \\ ${ }^{6}$ Department of Radiation Oncology, Mahavir Cancer Sansthan, Patna, India
}

Received January 12, 2015; Revised March 24, 2015; Accepted March 31, 2015; Published Online June 02 , 2015

\section{Original Article}

\begin{abstract}
Purpose: It has been seen that there is a clinically significant variation in the volume calculated across different planning systems for the same digital imaging and communication (DICOM) contours. Aim: The purpose of this study is to investigate the difference in volumes of organs at risk when the structure sets were exported from the Eclipse ((Palo Alto, USA Version 10.0) to XIO CMS (Electa, Crawley, UK Version 4.40.00) treatment planning system (TPS) and identify how the differences occur. Methods: We prospectively analyzed the volumes of organs at risk from computerized tomography (CT) data of 54 patients. Head and neck and brain tumors were taken for this study and contoured on Eclipse treatment planning system (TPS) after importing images from CT. These contoured images were then exported using radiotherapy DICOM transfer facility to XIO CMS planning system and compared the contoured volumes with Eclipse TPS structured volumes. Results: Our analysis showed that the differences in calculated volumes of the contours for the patients between the two planning systems can be large. Mixed results are shown for different organs with the absolute volume difference ranging from $-0.25 \mathrm{cc}$ to $319.73 \mathrm{cc}$. These results clearly shown that the two TPS interprets the contours differently when calculating the volume, and there is a closer match with the theoretical calculated volumes with XIO CMS calculated volumes. Conclusion: Observed discrepancies were consistent between the two planning systems. This impact of contouring variability could play a role on plan quality metrics which is used as criteria for clinical trial protocol compliance.
\end{abstract}

Keywords: Organ at Risk; Volume Variations; Treatment Planning Systems; Digital Imaging and Communication Transfer

\section{Introduction}

Computerized treatment planning systems ${ }^{1-2}$ have occupied major space in the field of radiation oncology. Software technology in Treatment planning systems made it possible to gather, organize and synthesize the large volume of data to provide input for more complex descriptions of target and organs at risk.

In commercial treatment planning system the treatment planner scans through the two dimensional (2D) anatomy slice by slice in all the three planes( axial, sagittal, coronal) and then contours gross tumor volume (GTV), clinical target volume (CTV) and organs at risk (OAR) on each of these slices individually either by manual or auto contouring methods. These are represented in three dimensions (3D) ${ }^{3}$ with the help of segmentation software.

Dose volume histogram (DVH) introduced by authors ${ }^{3-4}$ plays a major role in planning evaluation ${ }^{5}$. The advances in imaging and radiotherapy techniques have increased the complexity of radiotherapy treatment planning with improved dose conformity to the target structures while reducing the dose to surrounding normal structures and organs at risk. The modern techniques like intensity modulated radiotherapy (IMRT), image guided radiotherapy (IGRT) and volumetrically modulated arc therapy (VMAT) require an in depth analysis of isodose distributions for tumor control probability 
(TCP), normal tissue complication probability (NTCP) ${ }^{6-7}$ and conformity indices (CI).

The evaluation TCP, NTCP and CI depend on DVH analysis. The accuracy of dose volume histogram depends on how well the target volumes and the OARs are delineated by the physician and also on the accuracy of calculation algorithms of planning systems. It has already been observed in earlier studies that there is a significant difference between the volumes calculated by different planning systems. ${ }^{8}$ This variation is because of different contouring algorithms by different planning systems. If we consider regular radiotherapy flowchart in a department the computerized radiotherapy treatment planning system shares the maximum portion of it right from the beginning of the patient downloading from DICOM transferred images to the plan evaluation and exporting. So the total outcome of radiotherapy results depend on the basic parameter about the accuracy of OAR volume (cc) calculated by the 3D TPS.

A lot of importance has been given to the contouring guidelines for target volumes and regarding inter and intra observer variations. This issue of variations in target volume across the treatment planning systems has been thoroughly addressed in a number of publications. ${ }^{9}$ However, till the date no systematic study has been conducted towards the implications in the contouring of organs at risk (OAR) 10-11 among different planning systems. It is important to add here that delineation and dose to OARs should be given sufficient priority when advance techniques of radiotherapy like IMRT, IGRT and VMAT aim to deliver higher doses to tumor volume, keeping the OAR doses as low as reasonably achievable. A systematic study was conducted towards delineation of OAR volume when the structure sets were transferred from Eclipse (Version 10.0) to XIO CMS (Version 4.40.00) planning systems and the results were analyzed to see the implications of using two different planning systems. This paper presents the results of this study.

\section{Methods and Materials}

\section{Image acquisition}

Fifty four patients of Head and Neck cancers and brain tumors treated by radiotherapy in the last two years were recruited for this study. Computed tomography (CT) data set with helical scans of $3 \mathrm{~mm}$ slice widths, $120 \mathrm{kVp}, 512 \times 512$ mega pixels were taken for these patients on Philips big bore CT. The length of the scan for head and neck was from the tip of the brain to the shoulder level up to thoracic D5 rib except for one with positron emission tomography (PET) CT done up to abdomen. For brain cases, CT scans were taken from the apex of the brain to till neck C6 level.
From the CT work station the images were transferred through Digital Imaging and Communication (DICOM) network to the eclipse contouring work station. All the relevant normal organs and critical structures were contoured by the radiation oncologists as shown in Figure 1. Right and left orbits, right and left lenses, right and left optical nerves, optic chiasm, pituitary, brain stem, right and left parotids, mandible, oral cavity and planning organ at risk( Prv (oc + pit)) for chiasm and pituitary together were considered for this analysis.
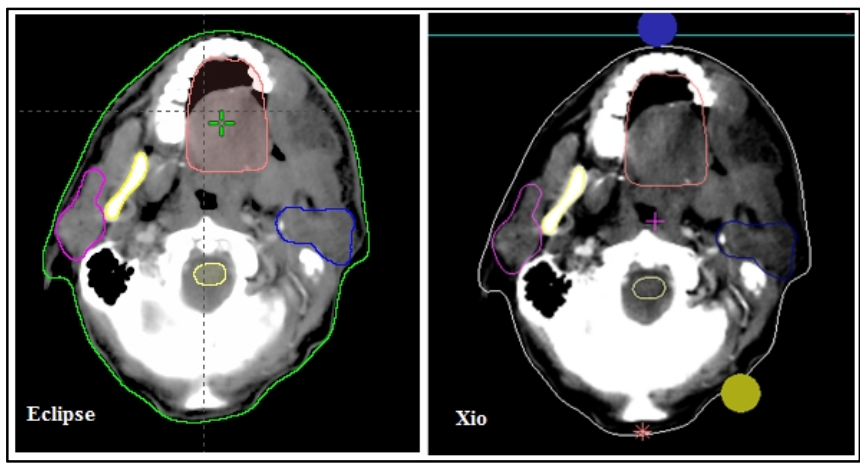

FIG. 1: Represents the contour delineated on Eclipse and dicom transferred contours on Xio CMS.

CT study sets of each patient along with the contoured structures were post processed and cross verified by another radiation oncologist. Volumes of all the critical structures were noted in cubic centimeters (cc). Through DICOM networking all the CT data with respective radiotherapy (RT) structures sets were transferred to Xio CMS planning system and the respective volumes of critical structures were noted for accounting inter TPS transfer variability. Finally the eclipse contoured volumes were evaluated and compared with the Xio CMS TPS transferred volumes. Comparisons were done for all critical structures. In our study we have chosen 15 critical structures and patient body. ICRU 62 guide lines were used to delineate the contours. ${ }^{12}$ The paired $t$-test was used to compare all the organs at risk volumes between the planning systems with eclipse as the reference system.

\section{Results}

The measured OAR volumes from the smallest volume (viz lens and chiasm) to the highest volume (viz body) were in the range of $0.01 \mathrm{cc}$ to 50, $000 \mathrm{cc}$ respectively. In our analysis, the measured volumes were categorized as less than 1cc and greater than 1ccvolumes for interpretation as shown in Table 1 and 2.

Table 1 and 2 summarizes the average volume of each of the organ contoured on Eclipse planning system and the Xio CMS measured volumes with mean absolute difference and standard deviation and $p$ value for paired t test. 
TABLE 1: Summary of OAR volumes greater than $1 \mathrm{cc}$.

\begin{tabular}{|c|c|c|c|c|c|c|c|c|c|c|}
\hline MEAN & Body & Brainstem & $\begin{array}{l}\text { Spinal } \\
\text { cord }\end{array}$ & $\begin{array}{l}\text { PRV } \\
\text { (oc+pit) }\end{array}$ & Oral & Mandible & $\begin{array}{l}\text { Right } \\
\text { parotid }\end{array}$ & $\begin{array}{l}\text { Left } \\
\text { Parotid }\end{array}$ & $\begin{array}{l}\text { Right } \\
\text { Orbit }\end{array}$ & $\begin{array}{l}\text { Left } \\
\text { Orbit }\end{array}$ \\
\hline Number-N & 54 & 51 & 53 & 47 & 45 & 48 & 51 & 54 & 54 & 53 \\
\hline Eclipse in cc & 13235.94 & 21.7765 & 21.3019 & 5.434 & 31.5467 & 45.2208 & 21.2588 & 20.5685 & 6.5926 & 6.4736 \\
\hline Xio CMS in cc & 13350.29 & 22.3377 & 21.4953 & 6.0794 & 33.1171 & 47.8058 & 21.788 & 21.1685 & 6.9067 & 6.8191 \\
\hline $\begin{array}{l}\text { Mean absolute } \\
\text { difference in cc }\end{array}$ & 114.3581 & 0.5612 & 0.1934 & 0.6453 & 1.5704 & 2.585 & 0.5292 & 0.6 & 0.3141 & 0.3455 \\
\hline Std dev & 49.400 & 0.198 & 0.238 & 0.301 & 0.865 & 1.254 & 0.306 & 0.279 & 0.188 & 0.328 \\
\hline$P$ value & $0.000^{8}$ & $0.000^{8}$ & $0.000^{8}$ & $0.000^{8}$ & $0.000^{8}$ & $0.000^{8}$ & $0.000^{8}$ & $0.000^{8}$ & $0.000^{8}$ & $0.000^{8}$ \\
\hline
\end{tabular}

TABLE 2: Summary of OAR volumes less than $1 \mathrm{cc}$.

\begin{tabular}{lllllll}
\hline \hline MEAN & $\begin{array}{l}\text { Rt } \\
\text { lens }\end{array}$ & $\begin{array}{l}\text { Let } \\
\text { lens }\end{array}$ & $\begin{array}{l}\text { Right } \\
\text { ON }\end{array}$ & $\begin{array}{l}\text { Left } \\
\text { ON }\end{array}$ & Chiasm & Pituitary \\
\hline Number-N & 53 & 53 & 54 & 53 & 54 & 54 \\
Eclipse in cc & 0.117 & 0.117 & 0.3074 & 0.2874 & 0.4411 & 0.1259 \\
Xio CMS in cc & 0.17 & 0.1732 & 0.4683 & 0.4551 & 0.8604 & 0.1917 \\
Mean absolute & 0.053 & 0.0562 & 0.1609 & 0.1677 & 0.4193 & 0.0657 \\
difference in cc & & & & & & \\
Std dev & 0.037 & 0.036 & 0.094 & 0.098 & 0.176 & 0.046 \\
$P$ value & $0.000^{8}$ & $0.000^{8}$ & $0.000^{8}$ & $0.000^{8}$ & $0.000^{8}$ & $0.000^{8}$ \\
\hline \hline
\end{tabular}

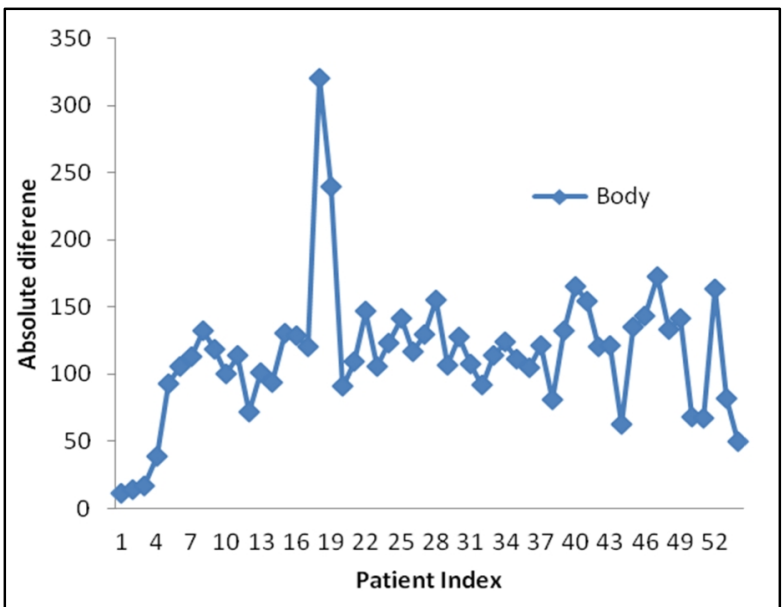

FIG. 2: Absolute differences in measured volumes by two treatment planning systems of 54 patients for body contours (cc).

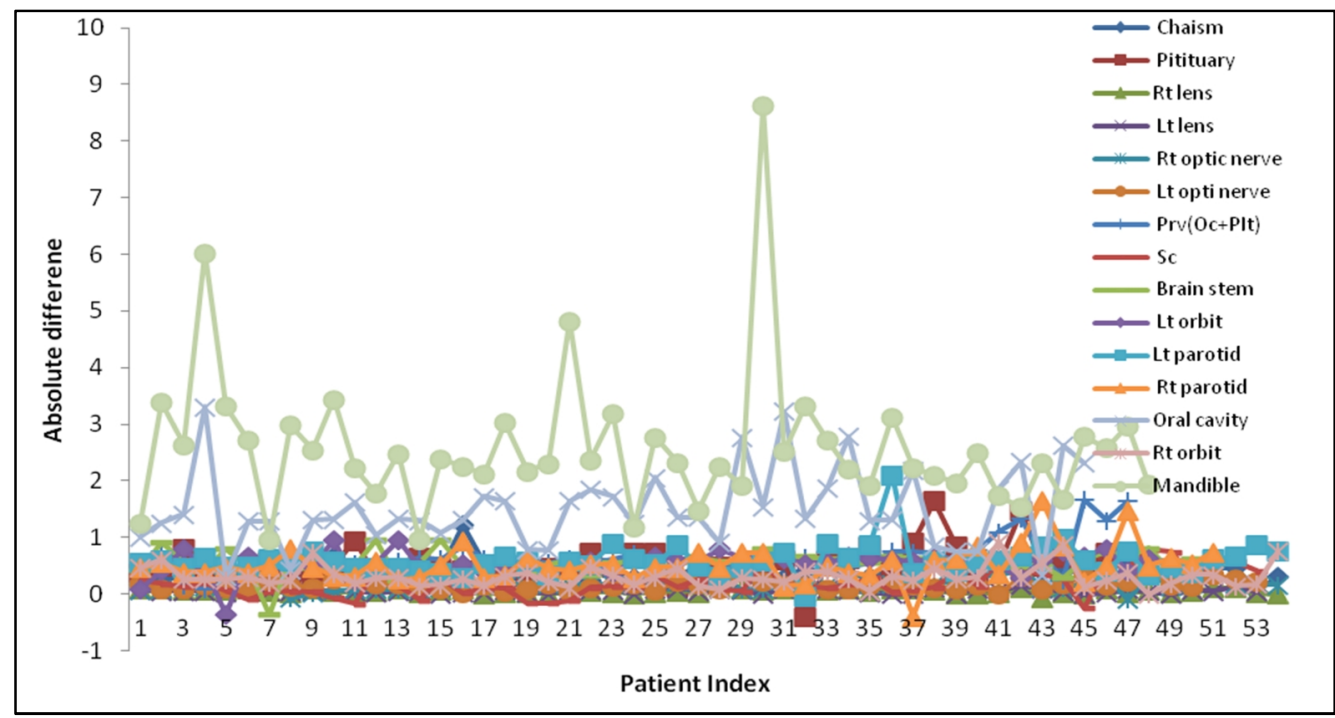

FIG. 3: Absolute differences in measured volumes by two treatment planning systems of 54 patients for OARs in cc. 


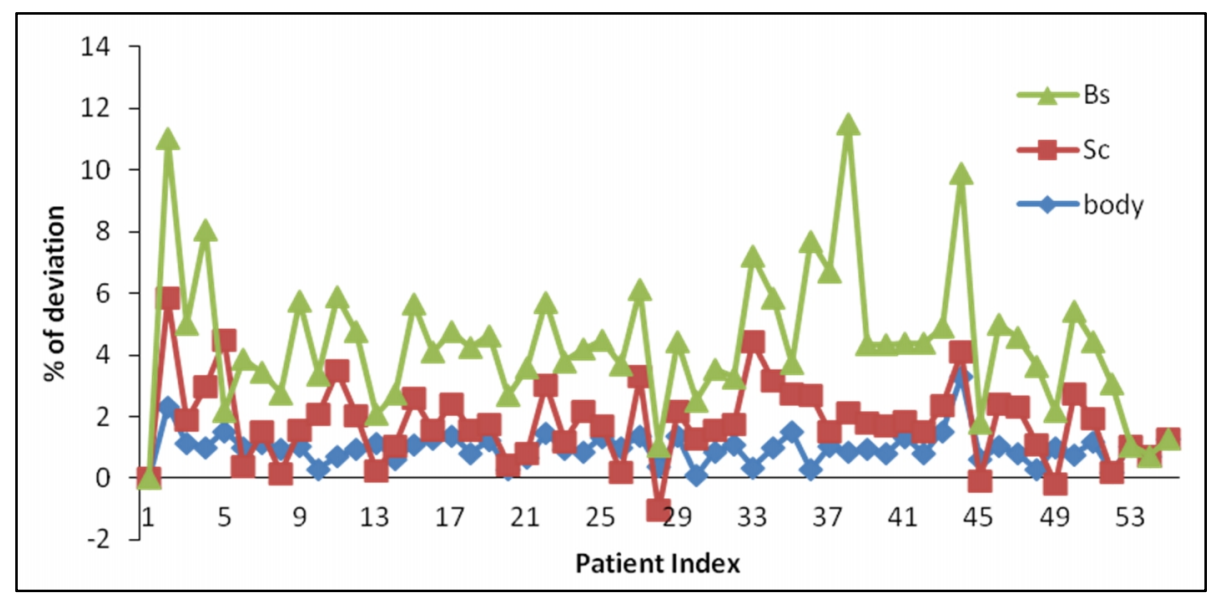

FIG. 4: Percentage (\%) deviation for OAR volumes of body spinal cord (SC) and brain stem (BS) in 54 patients.

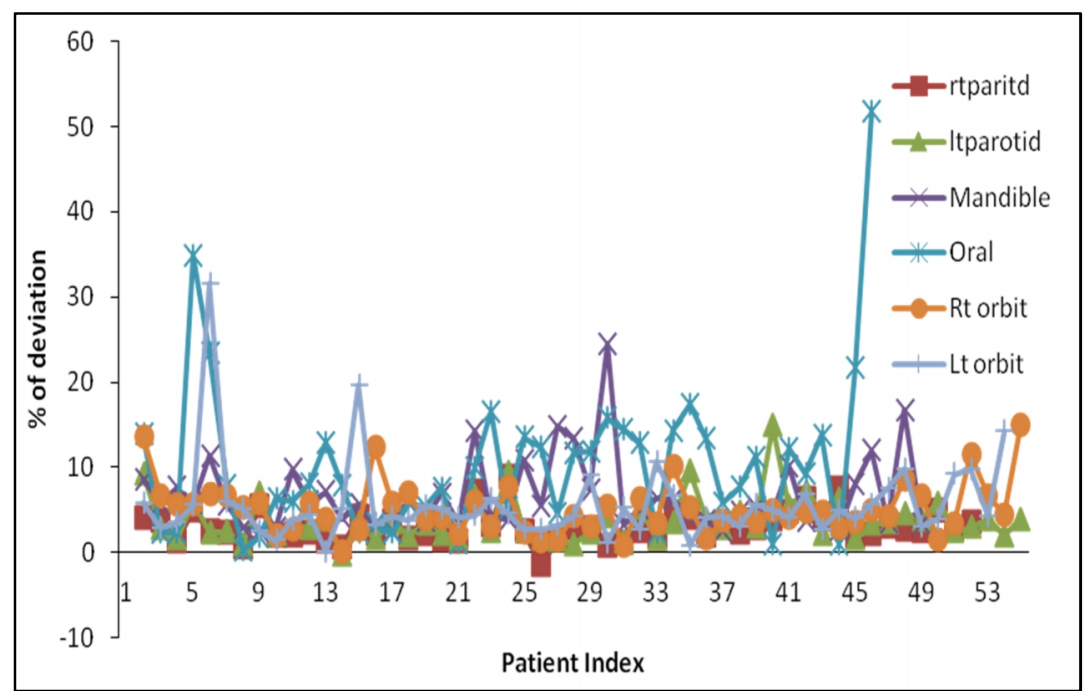

FIG. 5: Percentage (\%) deviation for OAR volumes of bilateral parotids, mandible oral cavity and bilateral orbits in 54 patients.

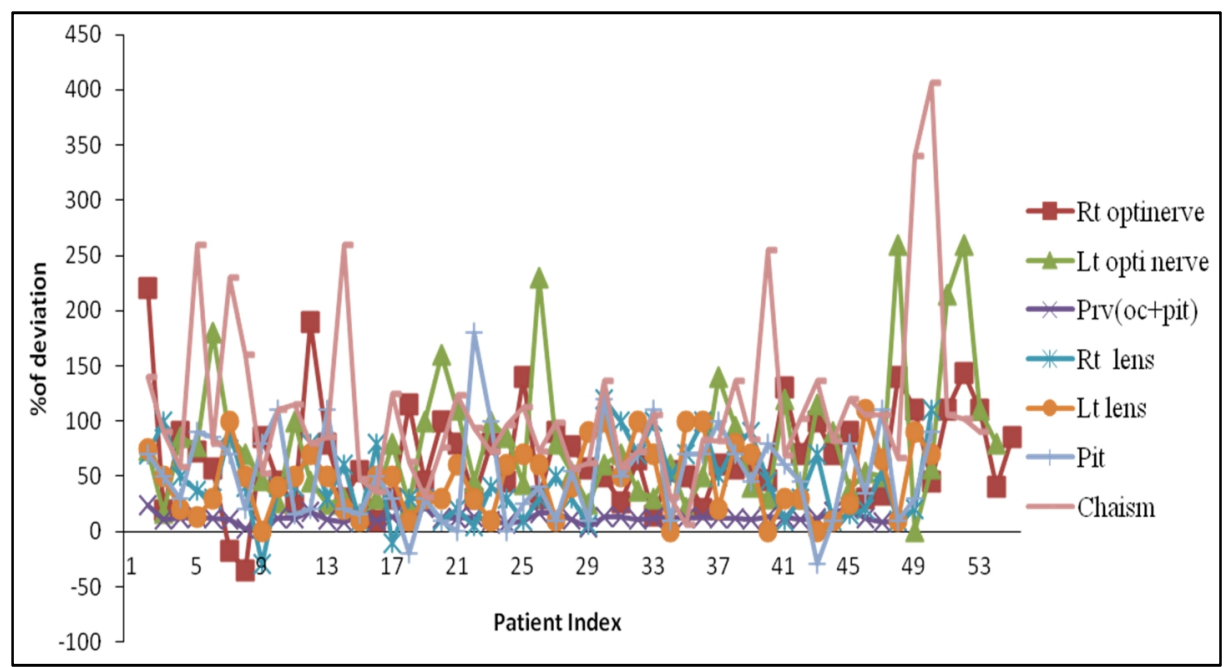

FIG. 6: Percentage (\%) deviation for OAR volumes of bilateral optic nerves and lens, chiasm, pituitary and PRV for (oc+pit) 54 patients. 


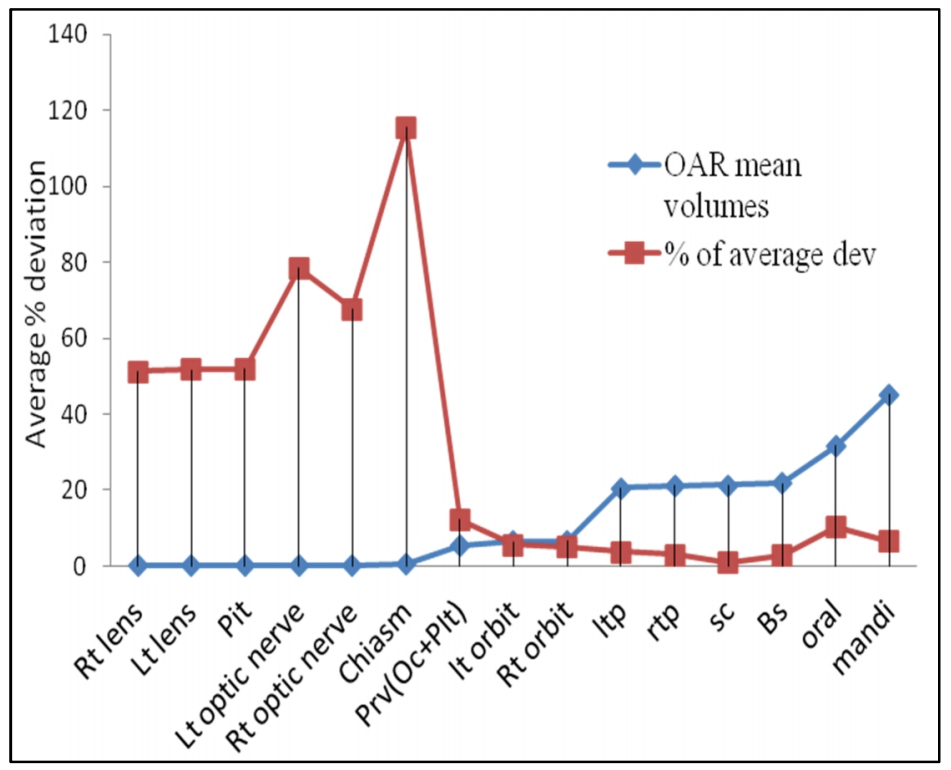

FIG. 7: Average of Percentage (\%) deviation for OARs.

Figures 2 and 3 shows the absolute volume differences of organs at risk computed in two planning systems. The Xio CMS TPS showed over estimation of volumes except spinal cord. In Body contour analysis, the difference in volume ranges from $11.3 \mathrm{cc}(0.29 \%)$ to $319.73 \mathrm{cc}(3.2 \%)$ [Figure 2-4]. In Brainstem and Eye contour analysis, Xio CMS showed volume difference with minimum and maximum \% variations of $1.28 \%(0.24 \mathrm{cc})$ to $4.9 \%(0.95 \mathrm{cc})$ and $0.00 \mathrm{cc}(0.0 \%)$ to $2.37 \mathrm{cc}(31.6 \%)$ respectively [Figure 3, 4 and 5]. Similarly, in lens contour analysis, difference in volumes ranges from -0.06 cc $(-30 \%)$ to 0.15 CC (250\%) [Figure 3-6]. In case of the spinal cord, Xio CMS TPS showed mixed results with the difference in volumes ranging from -0.25 cc $(-0.69 \%)$ to 0.79 cc (1.95\%) [Figure 3-4]. In mandible the observed difference in volume ranged from $0.96 \mathrm{cc}(2.82 \%)$ to $8.63 \mathrm{cc}(14.35 \%)$ [Figure 3-5].

From the Figure 4, 5 and 6, we can see that volumes with greater than $1 \mathrm{cc}$ has deviation from $-2.23 \%$ to $51.71 \%$ whereas for volumes less than 1cc, the deviations are from $-30.0 \%$ to $406.66 \%$. When we look at the graph of average $\%$ deviation in Figure 7 one can observe that for larger volume the graph is linear but for smaller volumes the graph shoots high indicating the conversion of contour points error at its highest.

\section{Discussion}

This study clearly shows the significant deviation in smaller volumes between the two planning systems. There are sufficient numbers of papers to address the issue of delineation of OARs and target volumes by a radiation oncologist using the advanced imaging techniques. Collier et al. ${ }^{13}$ has shown the contouring discrepancies between inter dosimetrists in manual contouring. Young et al. ${ }^{14}$ has discussed in their paper the difference of more than 15\% from PET contoured volumes on two different planning systems due to the difference between data formatting and display of contouring. In this paper they have separately contoured on each planning systems. Prabhakar et al. ${ }^{15}$ assessed on the variation of Tumor volume between different treatment planning systems. The tumor volume for normal IMRT and IGRT in head and neck is generally greater than $300 \mathrm{cc}$ but the OARs in this region ranges between $0.1 \mathrm{cc}$ (e.g. chiasm, optic nerves and lenses) to $10 \mathrm{cc}$ (e.g. orbits) and $30 \mathrm{cc}$ to $50 \mathrm{cc}$ ( e.g. brain stem, spinal cord and bilateral parotids). Our study mainly focuses on the elucidation of volumes by TPS during inter transfer of OAR volumes. Figure 7 clearly indicates that the shape and size of the organs could be one of the reasons for this variation as one can see the bilateral orbits; lens, pituitary and brain stem have almost spherical geometry whereas chiasm and optic nerves are irregular in shape.

It can be inferred that there is a difference in computing the volume between each treatment planning systems. In Medical imaging we reconstruct an organ or area of interests from a series of 2 dimensional slices. There are different algorithms to mark the boundaries of the same grey value (called as contouring) of an organ or region of interest. Every commercially available radiotherapy TPS use different types of contouring algorithms for defining the regions. Eclipse TPS uses shape based interpolation algorithm. ${ }^{16}$ In this method when the distance between the two slices is far more than the picture elements (pixels) an interpolation of grey values is done in between or the missing values to construct 3 dimensional volume. Whereas CMS Xio with a virtual grid size of 1 millimeter $(\mathrm{mm}) \times 1$ millimeter $(\mathrm{mm})$, measures the distance from the center of every cell outside the contour to the centre of every cell at the nearest point of contour to construct 3 dimensional volume by using rolling ball method. 
To detect the amount of variation between the two volumes we can use the mean distance to conformity as proposed by Jena et al. ${ }^{17}$ but this tool cannot be used for each transfer of data in a busy centre. Because of growing use of CT based treatment planning in advanced radiotherapy quality assurance (QA) of TPS becomes a necessity. There are a number of recommendations to carry out the QA of TPS in terms of dosimetric and non dosimetric tests comprehensively. ${ }^{18-19} \mathrm{But}$ it is difficult to choose, which test is appropriate and suitable for a given planning procedure and it is usually left onto the individual for taking such decisions as per the requirement of the technique. Craig et al. ${ }^{20}$ have performed QA for three different TPS using QUASAR phantom. However no consistent assessing techniques exist for comparing volumes interpreted by different planning systems.
Since the TPS showed variation for smaller organs, to access this variation pattern we created a set of discrete test volumes, by contouring on Eclipse TPS each with $0.5 \mathrm{~cm}, 1.0 \mathrm{~cm}, 1.5$ $\mathrm{cm}, 2.0 \mathrm{~cm}, 2.5 \mathrm{~cm}, 3.0 \mathrm{~cm}$ and $3.5 \mathrm{~cm}$ square shapes in a patient with images acquired on CT Phillips scanner. Care was taken to keep the slice separation of $3 \mathrm{~mm}$. All the test volumes were categorized in single, double and triple slices were noted separately for the assessment as shown in Table 3. These contoured structures were exported through DICOM transfer to the Xio CMS TPS for further analysis. We have calculated the theoretical volumes for our test volumes with $n$ and n-1 number of slices.

TABLE 3: Presents the values for standard volumes contoured on Eclipse and measured after transferred on CMS Xio from 0.5 to $3.5 \mathrm{~cm}$ squares with theoretical calculated values.

\begin{tabular}{|c|c|c|c|c|c|c|c|}
\hline \multirow{2}{*}{$\begin{array}{l}\text { Parameters } \\
\text { Square }\end{array}$} & \multicolumn{7}{|c|}{ Single slice volumes in cc } \\
\hline & 0.50 & 1.00 & 1.50 & 2.00 & 2.50 & 3.00 & 3.50 \\
\hline Contoured (Eclipse) & 0.01 & 0.05 & 0.16 & 0.35 & 0.69 & 1.14 & 1.77 \\
\hline Transferred (CMS) & 0.06 & 0.29 & 0.67 & 1.23 & 1.98 & 2.88 & 4.06 \\
\hline Theoretical volume (area*slice thickness * no of slices) & 0.08 & 0.30 & 0.68 & 1.20 & 1.88 & 2.70 & 3.68 \\
\hline Theoretical volume (area*slice thickness *no of slices-1) & 0.00 & 0.00 & 0.00 & 0.00 & 0.00 & 0.00 & 0.00 \\
\hline \multirow[t]{2}{*}{ Difference } & 0.05 & 0.24 & 0.51 & 0.88 & 1.29 & 1.74 & 2.29 \\
\hline & \multicolumn{7}{|c|}{ Double slices volume in cc } \\
\hline Square & 0.50 & 1.00 & 1.50 & 2.00 & 2.50 & 3.00 & 3.50 \\
\hline Contoured & 0.07 & 0.38 & 0.92 & 1.63 & 2.70 & 4.06 & 5.81 \\
\hline Transferred & 0.15 & 0.59 & 1.35 & 2.52 & 3.96 & 5.80 & 8.07 \\
\hline Theoretical volume (area*slice thickness ${ }^{*}($ no of slices)) & 0.15 & 0.60 & 1.35 & 2.40 & 3.75 & 5.40 & 7.35 \\
\hline Theoretical volume (area*slice thickness *no of slices-1) & 0.08 & 0.30 & 0.68 & 1.20 & 1.88 & 2.70 & 3.68 \\
\hline \multirow[t]{2}{*}{ Difference } & 0.08 & 0.21 & 0.43 & 0.89 & 1.26 & 1.74 & 2.26 \\
\hline & \multicolumn{7}{|c|}{ Triple slices volumes in cc } \\
\hline Square & 0.50 & 1.00 & 1.50 & 2.00 & 2.50 & 3.00 & 3.50 \\
\hline Contoured & 0.14 & 0.71 & 1.67 & 2.90 & 4.72 & 6.98 & 9.85 \\
\hline Transferred & 0.21 & 0.86 & 2.26 & 3.78 & 5.98 & 8.68 & 12.07 \\
\hline Theoretical volume (area*slice thickness *no of slices) & 0.23 & 0.90 & 2.03 & 3.60 & 5.63 & 8.10 & 11.03 \\
\hline Theoretical volume (area*slice thickness *no of slices-1) & 0.15 & 0.60 & 1.35 & 2.40 & 3.75 & 5.40 & 7.35 \\
\hline Difference & 0.07 & 0.15 & 0.59 & 0.88 & 1.26 & 1.70 & 2.22 \\
\hline
\end{tabular}

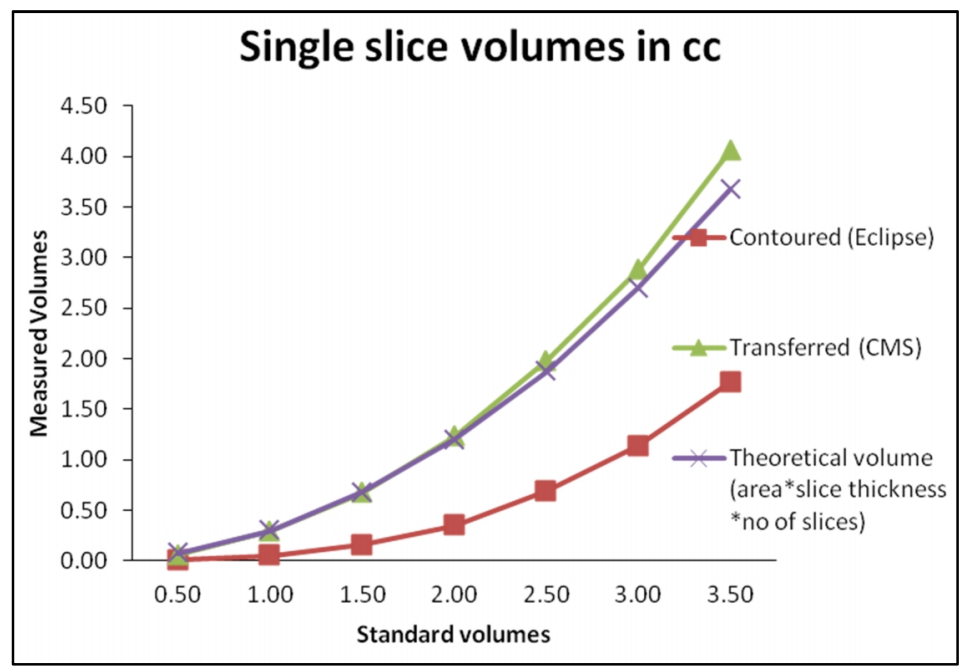

FIG. 8: Estimation of standard volumes by Xio CMS and Eclipse on single slice and the corresponding theoretical values. 


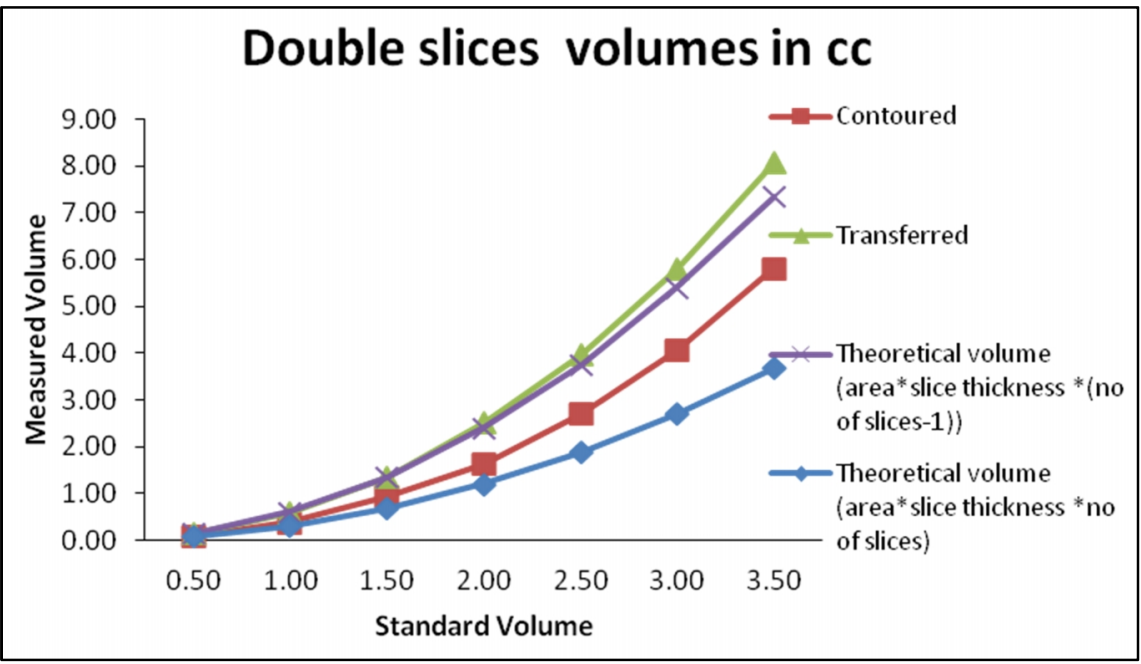

FIG. 9: Estimation of standard volumes by Xio CMS and Eclipse on double slice and the corresponding theoretical values.

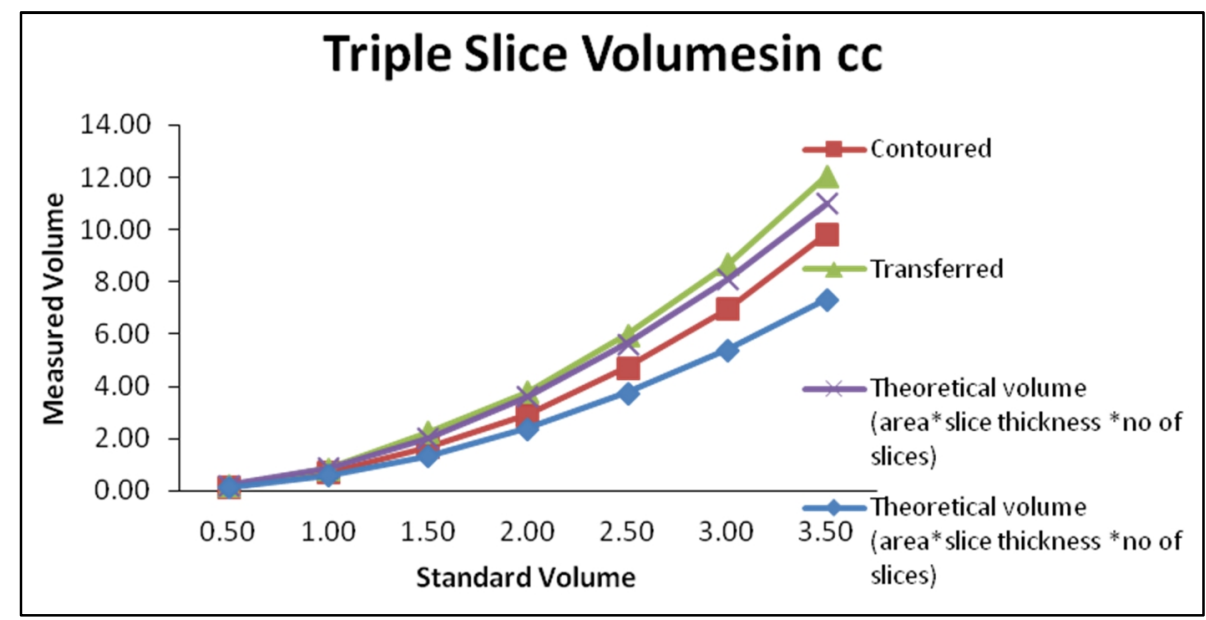

FIG. 10: Estimation of standard volumes by Xio CMS and Eclipse on triple slice and the corresponding theoretical values.

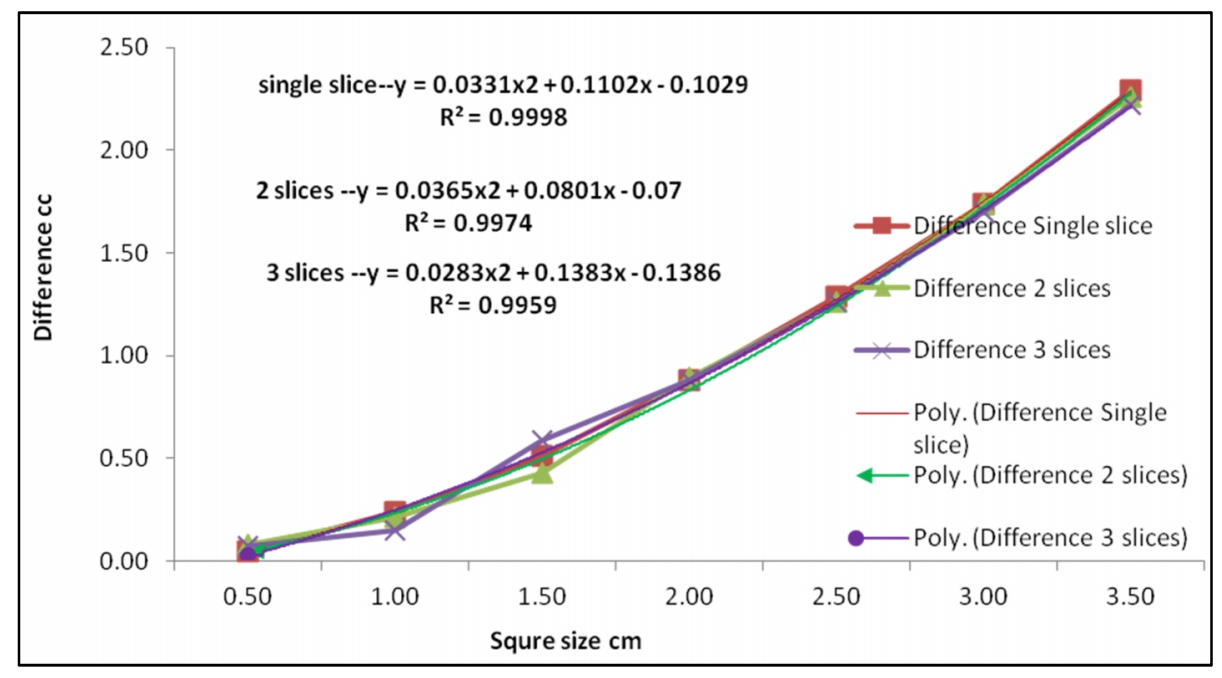

FIG. 11: Simple difference between the two TPS for standard volumes on slices. 


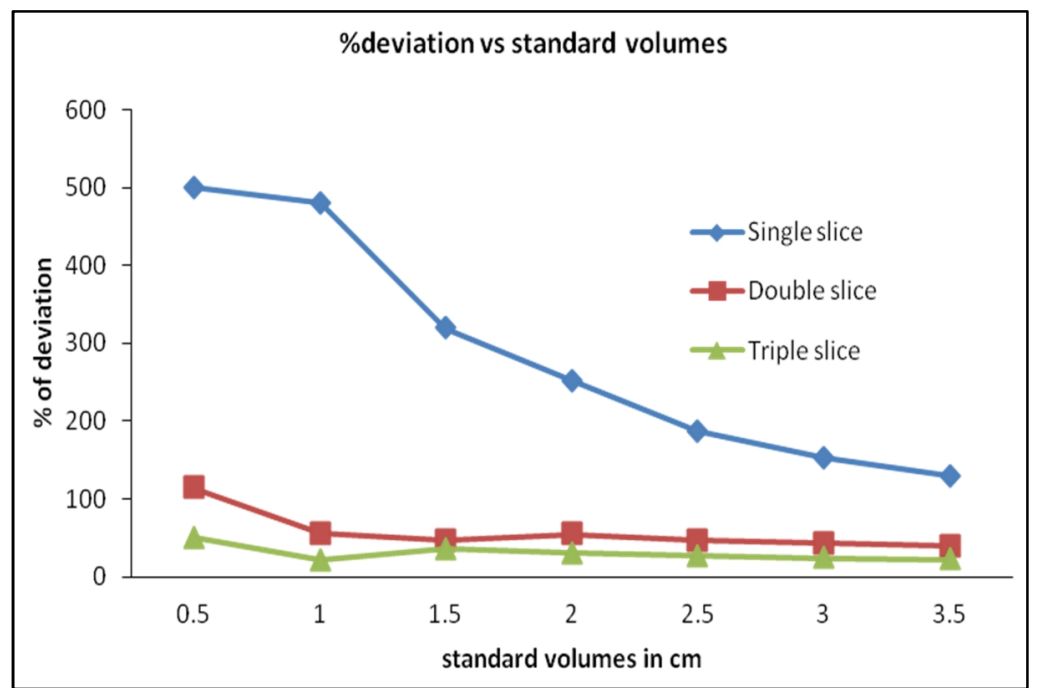

FIG. 12: Percentage (\%) of deviation between Eclipse measured and Xio CMS transferred for standard volumes.

From Figure 8, it is noted that the volumes estimated by Xio CMS TPS closely matches with the theoretical values on single slices. Similar behavior observed for the double and triple slices except that, when $\mathrm{n}-1$ slices are considered there is under estimation for the volumes from the original contoured slices. Figure 11 explains the difference graph with the consistent regression value following the linear relationship. A Graph of percentage of deviation versus standard volumes is shown in Figure 12. In single slice the deviation is higher for $0.5 \mathrm{~cm}$ square yielding $500 \%$ deviation to a volume of 3.5 m squares yielding $129.3 \%$ deviation lowest. Similarly, the differences in contours for double and triple slices range from $114.3 \%$ to $38.9 \%$ and $50 \%$ to $22 \%$ respectively. This variation is consistent among all the slices. So the variation is independent of the number of slices. Then it could be the kind of algorithm used to predict volume of interest and to some extent may be the size and shape of contour that may play some role. To identify the behavior of these different algorithms is out of scope for this paper.

A careful vigilance is required when one wants to fulfill the aim of maximum dose to targets with minimum dose to OARs volumes with proper dose volume recording in radiotherapy. The important point is we are utilizing the state of the art techniques like Computed tomography (CT), magnetic resonance imaging (MRI), ultra-sonography (US) and positron emission tomography (PET) ${ }^{21-22}$ for OAR segmentation with advance tools in TPS and each TPS has its own contouring algorithm. So if there are multiple treatment planning systems of different makes and models are available to the radiotherapy department individualized TPS QA becomes most important task to account for the inter TPS volume data transfer. ${ }^{23}$

\section{Conclusion}

Our analysis showed that the differences in calculated volumes of the structures for the patients between the two planning systems shows consistent results ranging from 0.25 cc to 319.73 cc. These results clearly indicate that Xio CMS TPS overestimated the volumes in comparison to Eclipse TPS but were comparable with calculated theoretical volumes. We also observed that the discrepancies were consistent between the two planning systems. So the overall accuracy to reproduce the same volumes differs between different planning systems. This indicates that when multiple planning systems are in use at single center or performing multicentre clinical plan comparison with different planning system the dose volume histogram ${ }^{24-25}$ directly depends on the volumes at comparison since smatter volumes end with bigger deviation.

\section{Conflict of interest}

The authors declare that they have no conflicts of interest. The authors alone are responsible for the content and writing of the paper.

\section{References}

1. Umegaki Y. Development of computer systems for radiotherapy of cancer. Jpn J Clin Oncol 2010; 40:e65-82.

2. Tsien KC. The application of automatic computing machines to radiation treatment planning. $\mathrm{BrJRa}$ diol 1955; 28:432-9.

3. Dowsett RJ, Galvin JM, Cheng E, et al. Contouring structures for 3-dimensional treatment planning. Int J Radiat Oncol Biol Phys 1992; 22:1083-8. 
4. Drzymala RE, Mohan R, Brewster L, et al. Dose-volume histograms. Int J Radiat Oncol Biol Phys 1991; 21:71-8.

5. Thompson RF. RadOnc: An R Package for Analysis of Dose-Volume Histogram and Three-Dimensional Structural Data. J Radiat Oncol Inform 2014: 6; 98-110.

6. Emami B, Lyman J, Brown A, et al. Tolerance of normal tissue to therapeutic irradiation. Int J Radiat Oncol Biol Phys 1991; 21:109-22.

7. Marks LB, Yorke ED, Jackson A, et al. Use of normal tissue complication probability models in the clinic. Int J Radiat Oncol Biol Phys 2010; 76:10-9.

8. Ackerly T, Andrews J, Ball D, et al. Discrepancies in volume calculations between different radiotherapy treatment planning systems. Australas Phys Eng Sci Med 2003; 26:91-3.

9. Zhu M, Bzdusek K, Brink C, et al. Multi-institutional quantitative evaluation and clinical validation of Smart Probabilistic Image Contouring Engine (SPICE) autosegmentation of target structures and normal tissues on computer tomography images in the head and neck, thorax, liver, and male pelvis areas. Int J Radiat Oncol Biol Phys 2013; 87:809-16.

10. Breunig J, Hernandez S, Lin J, et al. A system for continual quality improvement of normal tissue delineation for radiation therapy treatment planning. Int J Radiat Oncol Biol Phys 2012; 83:e703-8.

11. Nelms BE, Tomé WA, Robinson G, Wheeler J. Variations in the contouring of organs at risk: test case from a patient with oropharyngeal cancer. Int J Radiat Oncol Biol Phys 2012; 82:368-78.

12. International Commission on Radiation Units and Measurements: Prescribing, recording, and reporting photon beam therapy (supplement to ICRU report 50). Bethesda; ICRU; 1999.

13. Collier DC, Burnett SS, Amin M, et al. Assessment of consistency in contouring of normal-tissue anatomic structures. J Appl Clin Med Phys 2003; 4:17-24.

14. Young T, Som S, Sathiakumar C, Holloway L. An investigation into positron emission tomography contouring methods across two treatment planning systems. Med Dosim 2013; 38:60-5.
15. Prabhakar R, Rath GK, Haresh KP, et al. A study on the tumor volume computation between different 3D treatment planning systems in radiotherapy. J Cancer Res Ther 2011; 7:168-73.

16. Raya SP, Udupa JK. Shape-based interpolation of multidimensional objects. IEEE Trans Med Imaging 1990; 9:32-42.

17. Jena R, Kirkby NF, Burton KE, et al. A novel algorithm for the morphometric assessment of radiotherapy treatment planning volumes. Br J Radiol 2010; 83:44-51.

18. Van Dyk J, Barnett RB, Cygler JE, Shragge PC. Commissioning and quality assurance of treatment planning computers. Int J Radiat Oncol Biol Phys 1993; 26:261-73.

19. Fraass B, Doppke K, Hunt M, et al. American Association of Physicists in Medicine Radiation Therapy Committee Task Group 53: quality assurance for clinical radiotherapy treatment planning. Med Phys 1998; 25:1773-829.

20. Craig T, Brochu D, Van Dyk J. A quality assurance phantom for three-dimensional radiation treatment planning. Int J Radiat Oncol Biol Phys 1999; 44:955-66.

21. Lecchi M, Fossati P, Elisei F, et al. Current concepts on imaging in radiotherapy. Eur J Nucl Med Mol Imaging 2008; 35:821-37.

22. Kessler ML, Li K. Image Fusion for Conformal Radiotherapy. AAPM. 2001

https:/www.aapm.org/meetings/2001AM/pdf/7213 -95766.pdf

23. Swiss Society for Radiobiology and Medical Physics (SGSMP/SSRPM/SSRFM) SGSMP Recommendation No 7 Quality control of treatment planning systems for teletherapy 1997. ISBN 3-908125-23-5

24. Pyakuryal A, Myint WK, Gopalakrishnan M, et al. A computational tool for the efficient analysis of dose-volume histograms from radiation therapy treatment plans. J Appl Clin Med Phys 2010; 11:3013.

25. Ebert MA, Haworth A, Kearvell R, et al. Comparison of DVH data from multiple radiotherapy treatment planning systems. Phys Med Biol 2010; 55:N337-46. 